\title{
Retraction Note: Upregulation of the long non-coding RNA AFAP1-AS1 affects the proliferation, invasion and survival of tongue squamous cell carcinoma via the $\mathrm{Wnt} / \beta$-catenin signaling pathway
}

\author{
Ze-you Wang ${ }^{1}$, Min Hu' ${ }^{1}$, Min-hui Dai ${ }^{2}$, Jing Xiong ${ }^{2}$, Shuai Zhang ${ }^{3,4}$, Han-jiang Wu' ${ }^{4}$, Shan-shan Zhang ${ }^{4,5^{*}}$ and \\ Zhao-jian Gong ${ }^{4^{*}}$
}

Retraction note to: Molecular Cancer https://doi.org/10.1186/s12943-017-0752-2

The authors have retracted this article [1] because there are errors in Figures $4 \mathrm{a}$ and $4 \mathrm{~b}$. In these figures a number of cell field views are replicated between experimental groups:

In Figure $4 \mathrm{~b}$ the Scc-9/Mock panel replicates the Scc-9/shR-NC panel in Figure 4a

In Figure $4 \mathrm{~b}$ the CAL-27/Mock panel replicates the CAL-27/Mock panel in Figure 4a

In Figure $4 \mathrm{~b}$ the CAL-27/sHR-Nc panel replicates the CAL-27/sHR-Nc panel in Figure 4a

In Figure $4 \mathrm{~b}$ the CAL-27/shR-AFAP1-AS1 panel replicates the CAL-27/shR-AFAP1-AS1 panel in Figure 4a

The conclusions of this study are therefore not reliable. Zhaojian Gong, Hanjiang Wu, Shanshan Zhang, and Shuai Zhang agree with this retraction. Ze-you Wang, Min Hu, Min-hui Dai, and Jing Xiong agree with the retraction but did not respond to further correspondence about the retraction notice.

\section{Author details}

'Department of Laboratory Medicine, The Second Xiangya Hospital, Central South University, Changsha 410011, Hunan, China. ${ }^{2}$ Department of

Ophthalmology, Xiangya Hospital, Central South University, Changsha 410008, Hunan, China. ${ }^{3}$ Department of Clinical Medicine, Fujian Medical University, Fuzhou 350100, Fujian, China. ${ }^{4}$ Department of Oral and Maxillofacial Surgery, The Second Xiangya Hospital, Central South University, Changsha 410011, Hunan, China. ${ }^{5}$ Department of Stomatology, Xiangya Hospital, Central South University, Changsha 410008, Hunan, China.
Published online: 01 June 2019

\section{Reference}

1. Wang Z-y, Min H, Dai M-h, Xiong J, Zhang S, Han-jiang W, Zhang S-s, Gong $Z-j$. Upregulation of the long non-coding RNA AFAP1-AS1 affects the proliferation, invasion and survival of tongue squamous cell carcinoma via the Wnt/3-catenin signaling pathway. Mol Cancer. 2018;17:3 https://doi.org/ 10.1186/s12943-017-0752-2.

* Correspondence: zhangshanshan@csu.edu.cn;

gongzhaojian4458@csu.edu.cn

${ }^{4}$ Department of Oral and Maxillofacial Surgery, The Second Xiangya Hospital,

Central South University, Changsha 410011, Hunan, China

Full list of author information is available at the end of the article

(c) The Author(s). 2019 Open Access This article is distributed under the terms of the Creative Commons Attribution 4.0 International License (http://creativecommons.org/licenses/by/4.0/), which permits unrestricted use, distribution, and reproduction in any medium, provided you give appropriate credit to the original author(s) and the source, provide a link to the Creative Commons license, and indicate if changes were made. The Creative Commons Public Domain Dedication waiver (http://creativecommons.org/publicdomain/zero/1.0/) applies to the data made available in this article, unless otherwise stated. 